\title{
Coulisses
}

Revue de théâtre

1 | Printemps 1990

Varia

\section{Joseph Melcore, animateur du Théâtre Universitaire}

Joseph Melcore et Rédaction

\section{OpenEdition}

1 Journals

Édition électronique

URL : http://journals.openedition.org/coulisses/1555

DOI : 10.4000/coulisses. 1555

ISSN : 2546-9460

Éditeur

Presses universitaires de Franche-Comté

Édition imprimée

Date de publication : 1 avril 1990

Pagination : 41-43

ISSN : 1150-594X

\section{Référence électronique}

Joseph Melcore et Rédaction, « Joseph Melcore, animateur du Théâtre Universitaire », Coulisses [En ligne], 1 | Printemps 1990, mis en ligne le 04 juillet 2017, consulté le 25 octobre 2019. URL : http:// journals.openedition.org/coulisses/1555 ; DOI : 10.4000/coulisses.1555

Ce document a été généré automatiquement le 25 octobre 2019

Coulisses 


\title{
Joseph Melcore, animateur du Théâtre Universitaire
}

\author{
Joseph Melcore et Rédaction
}

\section{C. : Quelle est votre conduite de travail ?}

J.M. : La démarche de travail commence en octobre. Elle comprend toutes les phases de la création c'est-à-dire l'approche du jeu dramatique et les techniques. Ce travail dure à peu près un mois et demi, ce qui permet entre autres aux étudiants de mieux se connaître et de former un groupe. Après, nous abordons les textes du répertoire. À ce moment-là, nous faisons intervenir toutes les compétences de l'université, à savoir les professeurs. Ils prennent en charge une grande partie du travail dramaturgique. Par la suite, lorsque nous avons toute cette documentation, nous essayons de trouver des images pour la mise en scène.

C. : Comment sont sélectionnés les thèmes?

J.M. : Nous essayons de nous aligner sur tout ce qui se fait comme actions culturelles dans la région et dans la ville. Par exemple, pour le centenaire de la création de l'institut Pasteur, nous avons monté un spectacle (Le Laboratoire) qui portait à la fois sur Pasteur mais aussi sur le développement de la culture scientifique. En effet, il nous paraissait intéressant de travailler sur ce que l'on peut appeler le «théâtre scientifique ", c'est-à-dire de voir comment il était possible de faire passer la science par le théâtre. Même chose pour « Essence ", spectacle que nous avons organisé dans le cadre du centenaire de la création de l'automobile. Il faut souligner aussi que pour nos représentations nous faisons appel aux montages de façon à ce que tout le monde trouve sa place par rapport à ce désir de faire du théâtre, de jouer dans un spectacle. Nous ne faisons pas de sélection, autrement dit nous créons des spectacles où il n'y a pas de dichotomie entre personnages principaux, secondaires, etc. Chacun doit avoir sa place dans une partition qui est équitable. Pour cela, nous utilisons des textes de répertoire. Il nous arrive aussi de travailler sur des textes élaborés par des étudiants dans le cadre de l'un de leur cours intitulé « Écriture dramatique ».

C. : Comment sont répartis les rôles? Y a-t-il négociation? 
6 J.M. : La distribution passe par le désir des gens, mais il est vrai qu'au bout de deux mois, j'ai déjà une image, une petite idée sur la question. Je demande donc aux étudiants ce qu'ils ont envie de jouer et je leur fais moi aussi des propositions. Je pense que ce qui est important pour une personne, c'est de travailler des personnages qui ne sont pas le personnage prioritaire. Ce que je veux dire par là, c'est que chez chaque personne, nous avons tendance à retrouver un peu le même personnage, nous pouvons facilement le remarquer quand nous faisons des improvisations par exemple. Il est courant de reconnaitre le même personnage dans les mêmes improvisations sur des thèmes différents. Ce personnage prioritaire, il est à l'origine des étiquettes que l'on peut mettre sur un acteur: «Toi, tu as le physique du valet, alors tu joues le valet.» Nous, ce qui nous importe, c'est plus de retrouver une conviction de l'acteur. C. : Comment vous situez-vous par rapport à une école de théâtre?

8 J.M. : Nous faisons ici un travail d'animation, nous ne formons pas des acteurs. Donner des recettes pour que les gens sachent rire ou pleurer... ça ne m'intéresse pas. Nous essayons plutôt de trouver ce qui fait que chaque personne est unique c'est-à-dire de faire une mise en théâtre de toutes les possibilités de cette personne pour arriver à une forme d'acteur qui ne ressemble à rien, ou tout ou moins à autre chose que ce qui existe actuellement. C'est pour cela que je ne pose pas le problème en termes de bons ou mauvais acteurs mais en terme d'ACTEUR tout court, c'est-à-dire faire acte : venir sur scène, revendiquer une parole. On peut être émouvant même si on ne maîtrise pas parfaitement les techniques. Si on a vraiment envie de dire quelque chose, tous les moyens sont bons et l'on peut devenir ce bon acteur. Même si je fais de la mise en scène, je me considère plus comme un animateur de théâtre et dans ce sens, je ne choisis pas les acteurs.

9 C. : Vous jouez aussi parfois?

10 J.M. : Oui, parce que j'aime bien jouer dans les spectacles, mais ça, c'est toujours en fonction des places disponibles.

11 C'est drôle la question : « Pourquoi vous faites du théâtre? » En fait, on ne peut pas dire pourquoi. Le tout, il est vrai, c'est d'avoir ce plaisir, cette envie d'être sur scène. C'est plus retrouver le jeu spontané, le côté un peu enfant. Bien sûr, nous faisons des mises en scène où effectivement nous disons des choses que nous avons envie de dire mais la fable est un prétexte, même le thème, car je crois que le thème principal est LE THÉÂTRE.

12 Ce qui m'intéresse de montrer aussi, c'est pourquoi les gens y viennent, ce qui se passe aux répétitions, je veux dire tout le travail souterrain qui ne se voit pas forcément. Le théâtre ne se fait pas uniquement au moment de la représentation. Les répétitions, même si elles sont parfois longues et difficiles, nous font découvrir des choses, ne serait-ce que toutes les ressources d'un texte par exemple. C'est ce moment là qui est beau aussi. Tout ce cheminement est très intéressant et c'est ça qui fait la vie du spectacle.

C. : Le fait de jouer un spectacle une seule fois, au mois de juin, ce n'est pas un peu frustrant?

14 J.M. : Oui, certainement, mais il peut y avoir de bons côtés aussi parce que le problème de reprise est extrêmement compliqué. Le fait de revenir sur un texte qui a déjà été travaillé est très difficile.

15 Dario Fo, nous l'avons joué plusieurs fois mais nous ne sommes pas obligés de faire de la diffusion. Toutefois, la diffusion est aussi une étape dans la pratique théâtrale. Pour ce 
qui est des reprises, la motivation collective, c'est-à-dire la prise en charge du collectif par chaque individu, a une grande importance. Quand nous l'aurons perdue, ça sera la fin de ce spectacle.



Le laboratoire, juin 1987. École des beaux-arts de Besançon. 